\title{
Promotion of synaptogenesis and neural circuit development by exosomes
}

\author{
Jun Yamada, Shozo Jinno \\ Department of Anatomy and Neuroscience, Graduate School of Medical Sciences, Kyushu University, Fukuoka, Japan \\ Correspondence to: Shozo Jinno, MD, PhD. Department of Anatomy and Neuroscience, Graduate School of Medical Sciences, Kyushu University, 3-1-1 \\ Maidashi, Higashi-ku, Fukuoka 812-8582, Japan. Email: sjnno@med.kyushu-u.ac.jp. \\ Provenance: This is an invited article commissioned by the Section Editor Dr. Liuhua Zhou (Department of Urology, Nanjing First Hospital, Nanjing \\ Medical University, Nanjing, China). \\ Comment on: Sharma P, Mesci P, Carromeu C, et al. Exosomes regulate neurogenesis and circuit assembly. Proc Natl Acad Sci U S A 2019;116:16086-94.
}

Submitted Sep 03, 2019. Accepted for publication Sep 26, 2019.

doi: $10.21037 /$ atm.2019.09.154

View this article at: http://dx.doi.org/10.21037/atm.2019.09.154

Various mediators, such as neurotransmitters, gap junctions, cytokines, cell adhesion molecules, and growth factors, are involved in cell-to-cell communication in the brain (1). In particular, extracellular vesicles (EVs) have recently attracted interest as a novel type of intercellular communication mediator under physiological and pathological conditions (2). EVs represent a group of cellderived vesicles that are released into the extracellular milieu (3), and they are generally divided into three major types: apoptotic bodies (500-2,000 nm), microvesicles $(50-1,000 \mathrm{~nm})$, and exosomes $(40-200 \mathrm{~nm})$. Apoptotic bodies are vesicles produced from cells undergoing programmed cell death, and microvesicles arise through the direct outward budding and fusion of the plasma membrane (4). The term exosome was named to define EVs that released from the endosomal membrane (5). Exosomes first develop as intraluminal vesicles through the inward budding of the late endosomal membrane of multivesicular bodies (MVBs). These MVBs then directly fuse with the plasma membrane and release their contents outside the cells as exosomes (6). Exosomes are capable of transferring biologically active macromolecules, such as proteins, lipids, and nucleic acids (DNA, mRNA, microRNA, and long noncoding RNA), to other cells (7). Studies have also shown that exosomes bind to the membrane receptors of recipient cells, and deliver their contents through the fusion or hemi-fusion of the two membranes. Clathrin- and caveola-mediated endocytosis, phosphatidylserine-mediated phagocytosis, and micropinocytosis are also known to be involved in exosome- mediated signaling.

Homeostasis in the adult brain is affected by the biogenesis and molecular contents of exosomes (8). For instance, exosomes released by neurons in an activitydependent manner can be recaptured by other neurons (9). Neurotransmitter release also stimulates the secretion of exosomes from oligodendrocytes (10), and the internalization of oligodendrocyte-derived exosomes by neurons results in greater tolerance to oxidative stress (11). Neuron-derived exosomes are internalized by microglia, and this leads to an enhancement of the removal of degenerating neurites (12).

Exosomes are also involved in the pathophysiology of neurodegenerative diseases (13). For instance, exosomes promote intercellular prion protein transmission in prion diseases. Stimulation of the release of exosomes enhances the cell-to-cell transmission of prion infectivity (14). Exosomes can not only enhance the transfer of prions between different cell types but also facilitate their transmission between cells from different species. Protection of neurons through astrocyte-derived exosomes might depend on the transportation of prion proteins (15). An accumulation of exosomes has been found in the amyloid plaques in the brains of patients with Alzheimer's disease (16). Therefore, researchers have hypothesized that exosomes could provide a conducive environment for amyloidogenic fibril formation. In addition, exosomes are involved in the pathophysiology of Parkinson's disease via the exportation of $\alpha$-synuclein (17). In particular, $\alpha$-synuclein 
is secreted from neuronal cells in a calcium-dependent manner by exosomes, and this can impact the viability of neighboring neurons.

Growing evidence indicates that exosomes may be involved in the regulation of neurogenesis. Embryonic cerebrospinal fluid containing exosomes is known to promote neural stem cell amplification via the mammalian target of rapamycin complex 1 pathway (18). Exosomes also regulate the molecular components of neurogenic niches and maintain the balance between the proliferation and differentiation of neural stem/progenitor cells (NPCs) (2). Exosomes derived from induced NPCs (iNPCs) increase the proliferation of NPCs by activating the extracellular signal-regulated kinase pathway (19). Proliferation of NPCs is increased by microRNA-21a, which is rich in exosomes derived from iNPCs (20). These findings indicate the potent effects of exosomes on neurogenesis.

In a recent study, Sharma et al. examined the potential involvement of exosomes in synaptogenesis and neural circuit development (21). The authors began by asking if purified exosomes obtained from human induced pluripotent stem cell (hiPSC)-derived neurons might affect cell proliferation in human primary neural cultures in vitro. Recipient primary human neural cultures showed a more significant increase in cell numbers following treatment with exosomes derived from hiPSCs than with control exosomes. Early postnatal neurogenesis in the hippocampus of mice at postnatal day 4 was measured to examine whether exosomes may affect cell proliferation in the developing neural circuits in vivo. When purified exosomes obtained from rat primary neural cultures were delivered into the mouse lateral ventricle, they showed an increased density of newborn granule cells in the hippocampus. These observations indicate that exosomes may promote neurogenesis both in vitro and in vivo.

Rett syndrome is a rare neurodevelopmental disorder that is caused by mutations in the gene encoding the methyl-CpG-binding protein 2 (MECP2) (22). Deletion of the $M E C P 2$ gene in neurons is sufficient to cause a Rett-like phenotype in mice (23). MECP2 knockout mice exhibited delayed neuronal maturation and premature synaptogenesis in the cerebral cortex (24). Compared to control neurons, neurons derived from MECP2 loss of function (MECP2LOF) hiPSCs displayed lower number of synapses, decreased density of dendritic spines, reduced size of somata, and abnormal electrophysiological activity (25). Proteomic and bioinformatic analyses by Sharma et al. have shown that exosomes enclose proteins that interplay with complicated signaling networks affected by MECP2 mutation (21). Namely, the effects on neuronal maturation and synaptogenesis may be different between MECP2LOF and control exosomes.

Sharma et al. then examined whether alterations of specific exosomal proteins obtained from MECP2LOF donor cultures might reflect the cellular expression of the proteins on western blots (21). Interestingly, differences in cargo between MECP2LOF and isogenic control exosomes were generally irrelevant to their cellular expression. For instance, the protein levels of cadherin 2, calmodulin 1, flotillin, GAP43, and L1CAM were higher in MECP2LOF cells than in isogenic controls. By contrast, the expression levels of flotillin and GAP43 were reduced in MECP2LOF exosomes, whereas those of cadherin 2 and L1CAM were not affected. Thus, they could hypothesize that MECP2LOF might results in specific protein changes in exosomes and that exosome protein cargo is not a mere copy of the cellular proteins.

Treatment of human primary neural cultures with MECP2LOF exosomes did not affect the proliferation of NPCs and survival of newborn neurons; however, treatment with isogenic control exosomes promoted the proliferation of NPCs and enhanced the survival of newborn neurons (21). Interestingly, the proliferation of astrocytes was promoted by treatment with both isogenic control and MECP2LOF exosomes. The knock down of MECP2 (MECP2KD) by using $M E C P 2$ shRNA lentivirus decreased the total cell numbers in human neural cultures, and treatment with isogenic control exosomes counteracted the reduction in total cell numbers in MECP2KD human neural cultures. However, it should be noted that MECP2LOF exosomes do not have a dominant acute deleterious effect on cell proliferation and neuronal differentiation.

Sharma et al. also examined whether isogenic control exosomes might be able to rescue impaired synapses and neural circuits (21). To evaluate the effect of exosome administration on synaptogenesis, MECP2LOF hiPSCderived NPCs were grown in vitro for 6 weeks and exposed to control exosomes over 8 days. Neural cultures treated with control exosomes exhibited higher synaptic density than did cultures not treated with exosomes. To test whether exosome treatment affects the development of circuit assembly, neuronal firing in neurospheres treated with control exosomes was compared to that in neurospheres treated with MECP2LOF exosomes. Raster plots of spike trains showed that the firing rate in neurospheres exposed to MECP2LOF exosomes was low, 
while treatment with control exosomes increased the firing rate in neurospheres. Furthermore, aligned raster plots displayed more synchronized firing in neurospheres exposed to control exosomes than in those exposed to MECP2LOF exosomes. These results indicate that control exosomes enhance synaptogenesis in MECP2LOF hiPSC-derived NPCs in vitro, and suggest that control exosome treatments are capable of rescuing the development of neural circuits.

Overall, Sharma et al. developed a reductionist experimental paradigm and conducted multidisciplinary research on the role of exosomes in synaptogenesis and neural circuit development, although the absence of behavioral data may be a slight limitation of the paper. While a number of previous studies have reported that exosomes play a critical role in the maintenance of homeostasis in the adult brain, the study by Sharma et al. is novel as they have found that exosomes promote the development of neural circuits both in vitro and in vivo. In addition, they have demonstrated that isogenic control exosomes are capable of reversing pathological changes in neurons caused by MECP2LOF, whereas MECP2LOF exosomes neither rescue nor exacerbate neural circuit deficits resulting from the genetic deletion of MECP2. Future research focusing on the treatment with functional exosomes may have a huge clinical impact, especially if the exosomes can be optimized to rescue neural circuit deficits underlying neurodegenerative diseases.

\section{Acknowledgments}

The authors thank Editage (www.editage.jp) for English language editing.

\section{Footnote}

Conflicts of Interest: The authors have no conflicts of interest to declare.

Ethical Statement: The authors are accountable for all aspects of the work in ensuring that questions related to the accuracy or integrity of any part of the work are appropriately investigated and resolved.

\section{References}

1. Sharma P, Schiapparelli L, Cline HT. Exosomes function in cell-cell communication during brain circuit development. Curr Opin Neurobiol 2013;23:997-1004.
2. Bátiz LF, Castro MA, Burgos PV, et al. Exosomes as Novel Regulators of Adult Neurogenic Niches. Front Cell Neurosci 2016;9:501.

3. Saeedi S, Israel S, Nagy C, et al. The emerging role of exosomes in mental disorders. Transl Psychiatry 2019;9:122.

4. Akers JC, Gonda D, Kim R, et al. Biogenesis of extracellular vesicles (EV): exosomes, microvesicles, retrovirus-like vesicles, and apoptotic bodies. J Neurooncol 2013;113:1-11.

5. Johnstone RM, Adam M, Hammond JR, et al. Vesicle formation during reticulocyte maturation. Association of plasma membrane activities with released vesicles (exosomes). J Biol Chem 1987;262:9412-20.

6. Lee Y, El Andaloussi S, Wood MJ. Exosomes and microvesicles: extracellular vesicles for genetic information transfer and gene therapy. Hum Mol Genet 2012;21:R125-34.

7. Reza-Zaldivar EE, Hernandez-Sapiens MA, Minjarez B, et al. Potential Effects of MSC-Derived Exosomes in Neuroplasticity in Alzheimer's Disease. Front Cell Neurosci 2018;12:317.

8. Harting MT, Srivastava AK, Zhaorigetu S, et al. Inflammation-Stimulated Mesenchymal Stromal CellDerived Extracellular Vesicles Attenuate Inflammation. Stem Cells 2018;36:79-90.

9. Chivet M, Javalet C, Hemming F, et al. Exosomes as a novel way of interneuronal communication. Biochem Soc Trans 2013;41:241-4.

10. Frühbeis C, Fröhlich D, Krämer-Albers EM. Emerging roles of exosomes in neuron-glia communication. Front Physiol 2012;3:119.

11. Frühbeis C, Fröhlich D, Kuo WP, et al. Neurotransmittertriggered transfer of exosomes mediates oligodendrocyteneuron communication. PLoS Biol 2013;11:e1001604.

12. Bahrini I, Song JH, Diez D, et al. Neuronal exosomes facilitate synaptic pruning by up-regulating complement factors in microglia. Sci Rep 2015;5:7989.

13. Riva P, Battaglia C, Venturin M. Emerging Role of Genetic Alterations Affecting Exosome Biology in Neurodegenerative Diseases. Int J Mol Sci 2019. doi: 10.3390/ijms20174113.

14. Guo BB, Bellingham SA, Hill AF. Stimulating the Release of Exosomes Increases the Intercellular Transfer of Prions. J Biol Chem 2016;291:5128-37.

15. Guitart K, Loers G, Buck F, et al. Improvement of neuronal cell survival by astrocyte-derived exosomes under hypoxic and ischemic conditions depends on prion protein. 
Glia 2016;64:896-910.

16. Rajendran L, Honsho M, Zahn TR, et al. Alzheimer's disease beta-amyloid peptides are released in association with exosomes. Proc Natl Acad Sci U S A 2006;103:11172-7.

17. Emmanouilidou E, Melachroinou K, Roumeliotis T, et al. Cell-produced alpha-synuclein is secreted in a calciumdependent manner by exosomes and impacts neuronal survival. J Neurosci 2010;30:6838-51.

18. Feliciano DM, Zhang S, Nasrallah CM, et al. Embryonic cerebrospinal fluid nanovesicles carry evolutionarily conserved molecules and promote neural stem cell amplification. PLoS One 2014;9:e88810.

19. Ma Y, Wang K, Pan J, et al. Induced neural progenitor cells abundantly secrete extracellular vesicles and promote the proliferation of neural progenitors via extracellular signal-regulated kinase pathways. Neurobiol Dis 2019;124:322-34.

20. Ma Y, Li C, Huang Y, et al. Exosomes released from neural progenitor cells and induced neural progenitor cells regulate neurogenesis through miR-21a. Cell Commun Signal 2019;17:96.

21. Sharma P, Mesci P, Carromeu C, et al. Exosomes regulate neurogenesis and circuit assembly. Proc Natl Acad Sci U S A 2019;116:16086-94.

22. Leonard H, Cobb S, Downs J. Clinical and biological progress over 50 years in Rett syndrome. Nat Rev Neurol 2017;13:37-51.

23. Chen RZ, Akbarian S, Tudor M, et al. Deficiency of methyl-CpG binding protein-2 in CNS neurons results in a Rett-like phenotype in mice. Nat Genet 2001;27:327-31.

24. Fukuda T, Itoh M, Ichikawa $T$, et al. Delayed maturation of neuronal architecture and synaptogenesis in cerebral cortex of Mecp2-deficient mice. J Neuropathol Exp Neurol 2005;64:537-44.

25. Marchetto MC, Carromeu C, Acab A, et al. A model for neural development and treatment of Rett syndrome using human induced pluripotent stem cells. Cell 2010;143:527-39.

Cite this article as: Yamada J, Jinno S. Promotion of synaptogenesis and neural circuit development by exosomes. Ann Transl Med 2019;7(Suppl 8):S323. doi: 10.21037/ atm.2019.09.154 